2010-07

Targeting DLL4 in tumors shows

preclinical activity but potentially

significant toxicity.

$\mathrm{Li}, \mathrm{J}-\mathrm{L}$

http://hdl.handle.net/10026.1/10312

10.2217/fon.10.62

Future Oncol

All content in PEARL is protected by copyright law. Author manuscripts are made available in accordance with publisher policies. Please cite only the published version using the details provided on the item record or document. In the absence of an open licence (e.g. Creative Commons), permissions for further reuse of content should be sought from the publisher or author. 


\title{
Targeting DLL4 in tumors shows preclinical activity but potentially significant toxicity
}

\author{
Ji-Liang Li¹, Adrian M Jubb' \& Adrian L Harris' \\ Cancer Research UK Molecular Oncology Laboratories, Weatherall Institute of Molecular Medicine, University \\ of Oxford, Oxford OX3 9DS, UK \\ †Author for correspondence: Tel.: +44 1865222457 n. Fax: +44 1865222431 n ji-liang.li@imm.ox.ac.uk
}

Evaluation of: Yan M, Callahan CA, Beyer JC et al.: Chronic DLL4 blockade induces vascular neoplasms. Nature 463, E6-E7 (2010). Delta-like ligand 4 (DLL4) is a Notch ligand that is critical in the formation of a functional vascular network in tumors. Blockade of DLL4-mediated Notch signaling strikingly increases nonproductive angiogenesis, but significantly inhibits tumor growth in preclinical mouse models. Thus, DLL4 has emerged as an attractive target for cancer therapy. Anti-DLL4 antibodies have recently entered clinical trials. However, the potential toxic effects of anti-DLL4 are poorly understood. In this article, Yan et al. reported that chronic DLL4 blockade abnormally activates endothelial cells, causes pathological changes of multiple organs and induces vascular neoplasms. The findings need confirmation in further studies using different tumor-bearing animals but, nevertheless, raise important safety concerns regarding the use of anti-DLL4 agents and warrant monitoring for these effects in clinical trials for targeting DLL4.

\section{Summary of methods \& results}

To explore the therapeutic prospective of targeting Delta-like ligand 4 (DLL4), Yan et al. investigated the potential side effects of blocking the DLL4-mediated Notch pathway in three animal species in nontumor-bearing animals [1]. Treatment of adult mice with a DLL4-specific antibody for 3 weeks induced marked histopathological changes in the liver, including sinusoidal dilation and centrilobular hepatocyte atrophy. Blockade of Notch signaling by administration of an antagonistic soluble DLL4 (the extracellular domain of DLL4 fused to a human IgG1 Fc fragment), an anti-Notch1 inhibitory antibody, or the $\gamma$-secretase inhibitor dibenzazepine caused similar pathological changes in mouse liver, suggesting an important role of DLL4-initiated Notch signaling in liver. Taking advantage of crossreaction of the DLL4 antibody with DLL4 proteins from multiple species, the authors then extended their studies to Sprague-Dawley rats and cynomolgus monkeys (Macaca fascicularis). Intravenous injection of anti-DLL4 into rats at $10 \mathrm{mg} / \mathrm{kg}$ every 3 days for a total of five doses or monkeys at $3 \mathrm{mg} / \mathrm{kg}$ once a week for a total of eight doses produced similar liver histopathology.

Alterations in gene expression in the mouse liver were assessed 2 days after the administration of $10 \mathrm{mg} / \mathrm{kg}$ of anti-DLL4 antibody. Compared with phosphate-buffered salinetreated controls, anti-DLL4 treatment significantly increased the expression of several genes by 1.5 - to four-fold. These included the genes encoding endothelium-specific proteins EGFL7 (an extracellular matrix protein), Esam and LGALS1 (a galactoside-binding protein), as well as DNA replication/cell cycle regulation proteins MCM3 and MCM7.

Serum alanine aminotransferase levels (a marker of hepatocyte damage) showed a significant dose-related increase in rats treated with anti-DLL4 for 8 weeks at 1, 3, 10 or $30 \mathrm{mg} / \mathrm{kg}$. This was associated with morphological changes in the liver, including marked atrophy of centrilobular hepatic cords, dilation of centrilobular hepatic sinusoids and bile ductular proliferations.

In addition to liver, radiolabeled anti-DLL4 antibody was shown to distribute to other organs, including the bone marrow, spleen and lung of athymic nude mice. Prominent thymic atrophy was also observed in immunocompetent animals treated with the anti-DLL4 antibody. More importantly, ulcerating, subcutaneous tumors that were typically well circumscribed were observed in one out of 15 male rats dosed at $3 \mathrm{mg} / \mathrm{kg}$ and three out 15 animals at both 10 and $30 \mathrm{mg} / \mathrm{kg}$ for 8 weeks. These tumors showed numerous features of malignant vascular neoplasms, including freely anatomizing and branching vascular channels, focally infiltrating borders, necrosis, nuclear pleomorphism and a high proliferation fraction. Adjacent to the vascular spaces were pericytes that showed immunoreactivity for smooth muscle actin and desmin. In addition to subcutaneous neoplasms, vascular
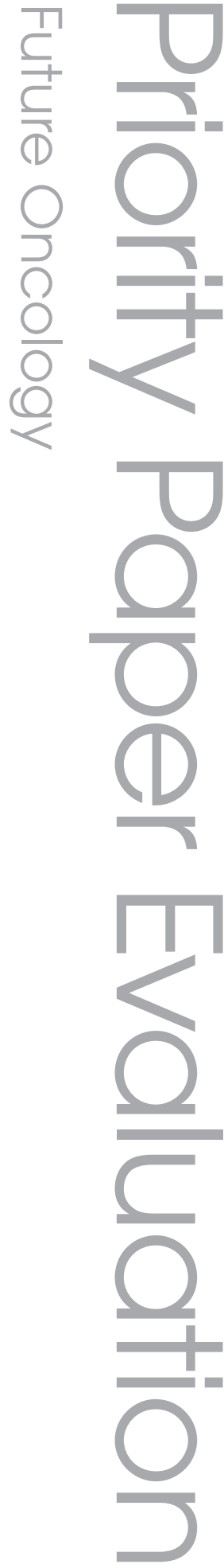

\section{Keywords}

w angiogenesis $=$ anti-DLL4

therapy $=$ DLL4 $=$ Notch

signaling = vascular neoplasm

- xenograft

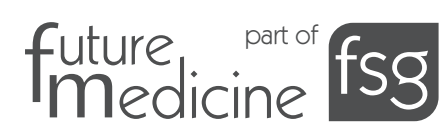


tumors containing regions of necrosis were infrequently found in the heart and lung of rats after anti-DLL4 antibody treatment for 8 weeks.

\section{Discussion}

Delta-like ligand 4 is predominantly expressed in arterial endothelial cells during embryogenesis $[2,3]$ and is markedly upregulated in the tumor vasculature of adult mammals [2,4-8]. Blockade of DLL4-mediated Notch signaling using an antiDLL4 antibody, soluble DLL4-Fc or a $\gamma$-secretase inhibitor has been shown to increase nonproductive angiogenesis, but significantly inhibit tumor growth in preclinical models [6,9-12]. Thus, DLL4 has emerged as an attractive target for cancer therapy [13], and anti-DLL4 antibodies have recently entered clinical trials. However, the potential side effects of blocking DLL4-mediated Notch signaling remain unclear.

Yan et al. reported that, unlike the $\gamma$-secretase inhibitor dibenzazepine, which indiscriminately blocks all Notch signaling, specifically targeting DLL4 with an anti-DLL4 antibody did not cause gastrointestinal toxicity [11] but produced marked histopathological changes in the liver of mice, rats and monkeys. These included centrilobular heptocyte atrophy, sinusoidal dilation and bile ductular proliferation [1]. As soon as 2 days after administration of the anti-DLL4 antibody, abnormal activation of endothelial cells occurred in mouse liver, as demonstrated by increased expression of three endothelium-specific genes and two genes concerned with DNA replication and cell cycle regulation. In keeping with these observations, treatment of rats with an anti-DLL4 antibody for 8 weeks was associated with a rise in the serum alanine aminotransferase levels, suggesting hepatocyte injury. Unfortunately, it is difficult to form a unifying hypothesis that the endothelial dysfunction seen in the mice is responsible for the hepatocyte toxicity seen in the rats. Complete experiments conducted within a single species are required to fully evaluate the clinical significance of these findings and the reversibility of both the endothelial dysfunction and hepatocyte injury.

No lethality was observed in mice and rats after 12 weeks of anti-DLL4 treatment [1]. However, DLL4 is abundantly expressed in the nervous system, the gastrointestinal system and the thymus during embryonic development [14], as well as in smaller arteries and microvessels in adult mice $[14,15]$. Prominent thymic atrophy was also evident in immunocompetent animals treated with anti-DLL4 antibody, consistent with the expression of DLL4 in thymic epithelial cells and the function of DLL4 in T-cell lineage commitment in thymus [16]. However, the presence of radiolabeled anti-DLL4 antibody in vessel-rich organs, such as the liver, bone marrow, spleen and lung (in athymic nude mice) was to be expected and does not necessarily imply toxicity [1]. Pathological and functional effects of chronic DLL4 blockade on other important organs, such as brain, kidney, lung and bone marrow, were not described.

Surprisingly, Yan et al. found that chronic DLL4 blockade was associated with the development of vascular neoplasms in rats in a doserelated manner. To be considered malignant, evidence is required to show that the tumors do not show involution on the discontinuation of DLL4 blockade and show aggressive local invasion and/or distant metastasis, which was not the case here. Nevertheless, the features described by Yan et al. would be consistent with a diagnosis of angiosarcoma in a human endothelial cell neoplasm. Indeed, the description of the cellular areas of these tumors is reminiscent of epithelioid differentiation, a feature of highly aggressive angiosarcomas. The distinction is important, as angiosarcomas that are not amenable to surgical excision have an exceptionally poor prognosis and those that are excised have a high recurrence rate. These observations were not reported in other species, such as mice and monkeys, and a histopathological study of the natural history of these tumors with continued therapy and on cessation of therapy is critical to inform their behavior.

We investigated the expression of DLL4 in several human cancers, including kidney, bladder, brain, colorectal and breast tumors [4-8]. To further explore the role of DLL4 in endothelial neoplasms, we examined the expression of DLL4 by immunohistochemistry in 16 human angiosarcomas [4]. Immunoreactivity for DLL4 was observed in the malignant cells of four out of seven breast, one out of one duodenal, one out of one liver, two out of five skin, one out of one pleural and zero out of one vaginal angiosarcomas. Immunoreactivity for DLL4 was strongest in the malignant endothelial cells of well-differentiated angiosarcomas showing prominent vascular channels (Figure 1A \& B). In several poorly differentiated areas with an epithelioid morphology (Figure 1C), the malignant cells were negative for DLL4 expression, in contrast to the strongly positive intervening vascular channels. Thus, DLL4 may have been a tumor suppressor gene in the etiology of some angiosarcomas that do not express DLL4. However, in other angiosarcomas that commonly express DLL4, DLL4 could be the direct target for therapy. 


\section{Conclusion \& future perspective}

Delta-like ligand 4 is strongly upregulated in tumor-associated endothelium and a small proportion of tumor cells in glioblastomas [6], colorectal adenomas/adenocarcinomas [5] and breast carcinomas [4]. Therefore, treatment with an anti-DLL4 antibody at a therapeutic level should preferentially bind to the tumor cells and tumor endothelial cells compared with normal tissue. It will be essential to investigate whether similar pathological changes would be produced by an anti-DLL4 antibody in tumor-bearing animals using doses that are optimally therapeutic, particularly studying organs such as the brain, liver, kidney, lung and bone marrow.

Chronic DLL4 blockade was associated with the development of subcutaneous neoplasms with uncertain malignant potential that have a vascular architecture [1]. It will be interesting to investigate whether these tumors are reversible after cessation of DLL4 blockade. It is not clear whether the development of vascular neoplasms is dependent upon the host gender and species. It would be informative to address the question using different strains of mice, rats and monkeys.

Single-agent antiangiogenic therapy, for example, anti-VEGF agents, shows comparatively poor activity and efficacy compared with combination therapy with cytotoxic agents in preclinical models and clinical trials [13]. Indeed, the combination of disrupted DLL4/Notch signaling with either blockade of the VEGF pathway or cytotoxic chemotherapy has shown a robust synergistic inhibition of tumor growth in preclinical mouse models [9,13]. Thus, disruption of both DLL4/Notch and VEGF pathways or use of a combination of anti-DLL4 and cytotoxic agents (e.g., irinotecan and paclitaxel) may have not only improved the therapeutic efficacy of DLL 4 blockade but may have also prevented the induction of vascular neoplasms. Using such combinations, particularly anti-VEGF therapy, in the model will be of major interest, to see if this prevents the development of the lesions.

Clearly, the reported toxic effects of this anti-DLL4 antibody in multiple organs and the induction of vascular neoplasms need to be confirmed in different tumor-bearing animals. This risk versus a clear antitumor benefit will need to be addressed in future trials. For the present, awareness of this issue should be taken into account in clinical monitoring of trials that are evaluating anti-DLL4 agents. However, it is important that the reversability or progression of these lesions is investigated.
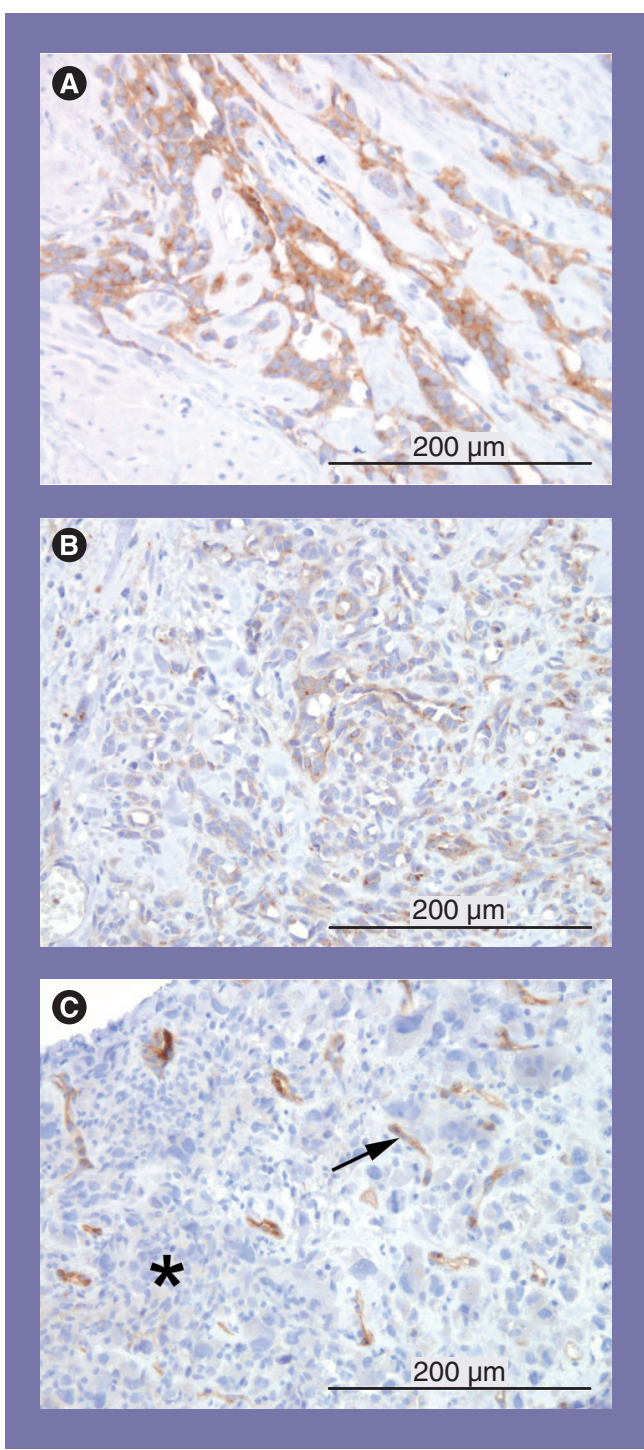

Figure 1. Immunohistochemistry showing DLL4 expression (brown membranous staining with diaminobenzidine against a blue nuclear hematoxylin counterstain).

(A) a well-differentiated breast angiosarcoma,

(B) a well-differentiated skin angiosarcoma and (C) an area of epithelioid morphology in a pleural angisarocoma (asterix: malignant epitheloid angiosarcoma cells that are negative for DLL4; arrow: intervening vascular channels that are positive for DLL4).

\section{Financial \& competing interests disclosure}

The authors have no relevant affiliations or financial involvement with any organization or entity with a financial interest in or financial conflict with the subject matter or materials discussed in the manuscript. This includes employment, consultancies, honoraria, stock ownership or options, expert testimony, grants or patents received or pending, or royalties.

No writing assistance was utilized in the production of this manuscript. 


\section{Priority Paper Evaluation Li, Jubb \& Harris}

\section{Executive summary}

\section{Study design}

- The inhibitors of Notch signaling that were investigated in the study were:

- Anti-Delta-like ligand 4 (DLL4) antibody (YW152F);

- Soluble DLL4 (the extracellular domain of DLL4 fused to human IgG1 Fc);

- Notch1-specific inhibitory antibody;

- The $\gamma$-secretase inhibitor dibenzazepine.

- The animals used in the study comprised of:

- Mice;

- Rats (Sprague-Dawley);

- Cynomolgus monkeys (Macaca fascicularis).

- The following dosing schedules were used in the study:

- Mice: intraperitoneal injection of anti-DLL4 antibody at $10 \mathrm{mg} / \mathrm{kg}$ twice per week for up to 8 weeks, phosphate-buffered saline as control;

- Rats: intravenous injection of anti-DLL4 antibody at $10 \mathrm{mg} / \mathrm{kg}$ every 3 days for a total of five doses; or at 1, 3, 10 and $30 \mathrm{mg} / \mathrm{kg}$, respectively, every 3 days for 8 weeks;

- Monkeys: intravenous injection of anti-DLL4 antibody at $3 \mathrm{mg} / \mathrm{kg}$ once per week for 8 weeks.

- The following outcome measures were tested and recorded:

- Histopathology: hematoxylin and eosin staining of tissues; immunochemical staining for proliferation (Ki67) and for pericyte markers, such as smooth muscle actin and desmin;

- Quantitative PCR for liver gene expression of Egfl7, Esam, Lgals1, Mcm3 and Mcm7;

- Liver-function tests by measuring serum alanine aminotransferase levels of rats;

- Formation of vascular tumors in multiple organs, including skin, heart and lung.

\section{Results}

- The following pathological changes occurred in the liver after anti-DLL4 treatment:

- Marked atrophy of centrilobular hepatic cords;

- Dilation of centrilobular hepatic sinusoids;

- Bile ductular proliferation.

- The following function changes occurred in the liver after anti-DLL4 treatment:

- Dose-related increase in alanine aminotransferase levels.

- The following molecule changes occurred in the liver after anti-DLL4 treatment:

- Upregulation of expression of the Egfl7, Esam, Lgals1, Mcm3 and Mcm7 genes.

- Vascular neoplasms in male rats were detected after anti-DLL4 treatment in the following locations:

- Within the skin;

- Rarely, lesions were found in the heart and/or lung tissue.

\section{Conclusion}

- Anti-DLL4 treatment caused pathological activation of endothelial cells.

- Chronic DLL4 blockade had the potential to disrupt normal organ homeostasis and produced significant pathology in multiple organs.

- Chronic DLL4 blockade induced vascular neoplasms.

\section{Bibliography}

Papers of special note have been highlighted as:

- of interest

1. Yan M, Callahan CA, Beyer JC et al.: Chronic DLL4 blockade induces vascular neoplasms. Nature 463(7282), E6-E7 (2010).

2. Mailhos C, Modlich U, Lewis J, Harris A, Bicknell R, Ish-Horowicz D: Delta4, an endothelial specific notch ligand expressed at sites of physiological and tumor angiogenesis. Differentiation 69(2-3), 135-144 (2001).

3. Shutter JR, Scully S, Fan W et al:: DLL4, a novel Notch ligand expressed in arterial endothelium. Genes Dev. 14(11), 1313-1318 (2000).
4. Jubb AM, Soilleux EJ, Turley H et al: Expression of vascular notch ligand Delta-like 4 and inflammatory markers in breast cancer. Am. J. Pathol. 76(4), 2019-2028 (2010).

5. Jubb AM, Turley H, Moeller HC et al: Expression of Delta-like ligand 4 (DLL4) and markers of hypoxia in colon cancer. Br. J. Cancer 101(10), 1749-1757 (2009).

6. Li JL, Sainson RC, Shi W et al.: Delta-like 4 Notch ligand regulates tumor angiogenesis, improves tumor vascular function, and promotes tumor growth in vivo. Cancer Res. 67(23), 11244-11253 (2007).
- Delta-like ligand 4 (DLL4) in mouse tumor models functioned as a negative regulator of tumor angiogenesis by reducing number of tumor vessels but acted as a positive driver for tumor growth by improving the structure and function of tumor vasculature; blockade of DLL4/Notch by soluble DLL4-mFc inhibited tumor growth.

7. Patel NS, Dobbie MS, Rochester M et al. Up-regulation of endothelial Delta-like 4 expression correlates with vessel maturation in bladder cancer. Clin. Cancer Res. 12(16), 4836-4844 (2006). 
8. Patel NS, Li JL, Generali D, Poulsom R, Cranston DW, Harris AL: Up-regulation of Delta-like 4 ligand in human tumor vasculature and the role of basal expression in endothelial cell function. Cancer Res. 65(19), 8690-8697 (2005).

9. Hoey T, Yen WC, Axelrod F et al: DLL4 blockade inhibits tumor growth and reduces tumor-initiating cell frequency. Cell Stem Cell 5(2), 168-177 (2009).

- Inhibition of human DLL4 in xenograft tumors, either alone or in combination with the chemotherapeutic agent irinotecan or paclitaxel, inhibited tumor recurrence and reduced cancer stem cell frequency in mouse models.

10. Noguera-Troise I, Daly C, Papadopoulos NJ et al.: Blockade of DLL4 inhibits tumor growth by promoting non-productive angiogenesis. Nature 444(7122), 1032-1037 (2006).

- DLL4/Notch blockade by soluble mDLL4-hFc or polyclonal anti-DLL4 antibody resulted in markedly increased nonproductive angiogenesis and decreased tumor growth of C6 glioma, mouse mammary tumor and HT1080 mouse models.

11. Ridgway J, Zhang G, Wu Y et al:: Inhibition of DLL4 signaling inhibits tumor growth by deregulating angiogenesis. Nature 444(7122), 1083-1087 (2006).

- Blockade of DLL4/Notch by DLL4-neutralizing humanized phage antibody (YW152F) inhibited tumor growth by deregulating angiogenesis in several tumor models but had no discernable effect on intestinal goblet cell differentiation.

12. Scehnet JS, Jiang W, Kumar SR et al.: Inhibition of DLL4-mediated signaling induces proliferation of immature vessels and results in poor tissue perfusion. Blood 109(11), 4753-4760 (2007).

- Soluble DLL4 significantly inhibited tumor growth but increased vascular density in human HT29 colon carcinoma and KS-SLK Kapsoi sarcoma xenografts.
13. Li JL, Harris AL: Crosstalk of VEGF and Notch pathways in tumor angiogenesis: therapeutic implications. Front. Biosci. 14, 3094-3110 (2009).

14. Benedito R, Duarte A: Expression of DLL4 during mouse embryogenesis suggests multiple developmental roles. Gene Expr. Patterns 5(6), 750-755 (2005).

15. Gale NW, Dominguez MG, Noguera I et al.: Haploinsufficiency of Delta-like 4 ligand results in embryonic lethality due to major defects in arterial and vascular development. Proc. Natl Acad. Sci. USA 101(45), 15949-15954 (2004).

16. Koch U, Fiorini E, Benedito R et al.: Delta-like 4 is the essential, nonredundant ligand for Notch1 during thymic T cell lineage commitment. J. Exp. Med. 205(11), 2515-2523 (2008). 